\title{
Local Percolation Probabilities for a Natural Sandstone
}

\author{
R. Hilfer ${ }^{1,2}$, T. Rage ${ }^{3}$ and B. Virgin ${ }^{3}$ \\ ${ }^{1}$ ICA-1, Universität Stuttgart, Pfaffenwaldring 27, 70569 Stuttgart \\ ${ }^{2}$ Institut für Physik, Universität Mainz, 55099 Mainz, Germany \\ ${ }^{3}$ Institute of Physics, University of Oslo, 0316 Oslo, Norway
}

\begin{abstract}
Local percolation probabilities are used to characterize the connectivity in porous and heterogeneous media. Together with local porosity distributions they allow to predict transport properties [1]. While local porosity distributions are readily obtained, measurements of the local percolation probabilities are more difficult and have not been attempted previously. First measurements of three dimensional local porosity distributions and percolation probabilities from a pore space reconstruction for a natural sandstone show that theoretical expectations and experimental results are consistent.
\end{abstract}


According to the mixing law of local porosity theory [1 10 the effective frequency dependent dielectric function $\varepsilon_{e}(\omega)$ of a heterogeneous mixture may be calculated by solving the integral equation

$$
\int_{0}^{1} \frac{\varepsilon_{C}(\omega ; \phi)-\varepsilon_{e}(\omega)}{\varepsilon_{C}(\omega ; \phi)+2 \varepsilon_{e}(\omega)} \lambda(\phi) \mu(\phi) d \phi+\int_{0}^{1} \frac{\varepsilon_{B}(\omega ; \phi)-\varepsilon_{e}(\omega)}{\varepsilon_{B}(\omega ; \phi)+2 \varepsilon_{e}(\omega)}(1-\lambda(\phi)) \mu(\phi) d \phi=0
$$

where

$$
\begin{aligned}
& \varepsilon_{C}(\omega ; \phi)=\varepsilon_{1}(\omega)\left(1-\frac{1-\phi}{\left(1-\varepsilon_{2}(\omega) / \varepsilon_{1}(\omega)\right)^{-1}-\phi / 3}\right) \\
& \varepsilon_{B}(\omega ; \phi)=\varepsilon_{2}(\omega)\left(1-\frac{\phi}{\left(1-\varepsilon_{1}(\omega) / \varepsilon_{2}(\omega)\right)^{-1}-(1-\phi) / 3}\right)
\end{aligned}
$$

In (1) the local porosity distribution $\mu(\phi)$ and the local percolation probability $\lambda(\phi)$ are geometrical input functions which will be defined shortly. The functions $\varepsilon_{1}(\omega)$, and $\varepsilon_{2}(\omega)$ are the frequency dependent dielectric functions of the pure materials. If $\varepsilon_{i}(\omega), i=1,2$ and $\mu(\phi), \lambda(\phi)$ are known then (1) gives a prediction for the effective dielectric function $\varepsilon_{e}(\omega)$.

Measurements of the local porosity distribution $\mu(\phi)$ are readily obtained from thin sections [6,8, 11]. Measurements of the local percolation probabilities $\lambda(\phi)$, on the other hand, are more difficult, and have not been reported. The main purpose in this paper is to present preliminary measurements of local percolation probabilities for a natural sandstone specimen.

Define $\mathbb{P}$ to be the pore space of a two component porous medium. The complement of $\mathbb{P}$ in $\mathbb{R}^{3}$ is called the matrix space $\mathbb{M}$. More generally $\mathbb{P}$ and $\mathbb{M}$ may represent the two components in a heterogeneous medium. The local porosity $\phi(\mathbb{K})$ measured within a measuring region $\mathbb{K}$ is defined by

$$
\phi(\mathbb{K})=\frac{V(\mathbb{P} \cap \mathbb{K})}{V(\mathbb{K})}
$$

where $V(\mathbb{K})$ is the volume of a set $\mathbb{K}$. Let $\mathbf{g}_{i} \in \mathbb{Z}^{3}$ denote the lattice vectors of a simple cubic lattice, and let 


$$
\mathbb{K}(\mathbf{r}, L)=\left\{\mathbf{r}^{\prime} \in \mathbb{R}^{3}:\left|r_{i}^{\prime}-r_{i}\right| \leq L / 2, i=1,2,3\right\}
$$

denote a cube of sidelength $L$ centered at $\mathbf{r}$.

Given the notation introduced above the local porosity distribution may be defined as [10]

$$
\mu(\phi, L)=\lim _{M \rightarrow \infty} \frac{1}{M} \sum_{i=1}^{M} \delta\left(\phi-\phi\left(\mathbb{K}\left(L \mathbf{g}_{i}, L\right)\right)\right)
$$

if the limit exists. Here $\delta(x)$ is the Dirac $\delta$-function. In practice the sample is finite, and hence the limiting process terminates after a finite number $M$ of measurement cells. The resulting histogram is used as an approximation for $\mu$.

Local porosity distributions quantify the fluctuations in volume fractions. To characterize the transport properties,however, it is necessary to quantify the degree of connectedness of the porous medium. Two points inside the pore space $\mathbb{P}$ are called connected if there exists a path entirely within the pore space that connects the two points. A cubic measurement cell $\mathbb{K}_{j}$ in a cubic partitioning is called percolating in the $x$-direction if there exists a path within $\mathbb{P} \cap \mathbb{K}_{j}$ connecting those two opposite faces of $\mathbb{K}_{j}$ that are perpendicular to the $x$-direction. Percolation in the $y$ - or $z$-direction is defined analogously. The local percolation probability $\lambda_{x}(\phi, L)$ in the $x$-direction is defined as the fraction of measurement cells that are percolating in the $x$-direction and have a local porosity $\phi$. The local percolation probabilities $\lambda_{y}(\phi, L)$ and $\lambda_{z}(\phi, L)$ are defined analogously.

To measure $\mu(\phi, l)$ and $\lambda(\phi, L)$ in practice it is necessary to reconstruct the three dimensional pore space $\mathbb{P}$ for digital processing. This was done by the method of serial sectioning for a Savonnier oolithic sandstone specimen [12,13]. This type of sandstone exhibits oomoldic porosity with ellipsoidal pores and grains of sizes between $200 \mu \mathrm{m}$ and $300 \mu \mathrm{m}$ [12]. After filling the stone with a coloured epoxy resin the specimen was cut and polished. The polished surface was photographed before removing another layer of material parallel to first 
surface. The second surface was then polished and photographed before iterating the procedure. A total of 99 sections was prepared. The distance between consecutive planes was about $10 \mu \mathrm{m}$. Each photograph represented an area of roughly $2 \mathrm{~cm} \times 2 \mathrm{~cm}$. The photographs were scanned and thresholded into a binary image with $1904 \times 1904$ pixels. This corresponds to a resolution within each image of roughly $10 \mu \mathrm{m}$. Figure 1 shows a typical binary image from within one of the 100 planes measuring roughly $1 \mathrm{~cm}$ along each side.

The family of three dimensional local porosity distributions $\mu(\phi, L)$ for this sample is obtained straightforwardly. The results are displayed in Figure 2 for various L. Ref. [9] has investigated the question to what extent the three dimensional local porosity distribution can be measured from two dimensional sections. While this is not generally possible, it was found that a two dimensional measurement combined with an appropriate rescaling of the length $L$ gives good results.

A preliminary measurement of the local percolation probabilities has also been carried out for the oolithic sandstone specimen. The results are shown in Figure 3 for cubic measurement cells of size $L=32$. The curve $\lambda_{x}(\phi ; 32)$ is shown in black, the curve $\lambda_{y}(\phi ; 32)$ is shown in medium gray, and the curve $\lambda_{z}(\phi ; 32)$ is shown in light gray. The local porosity distribution for $L=32$ is reproduced as the inset.

While the data show much scatter these preliminary estimates for the local percolation probabilities indicate that the sandstone has anisotropic connectivity. The same trend is observed for smaller as well as for larger values of $L$, although the strength of the anisotropy appears to change slightly.

The data are in agreement with the theoretical expectation that the local percolation probabilities should increase monotonically from 0 to 1 . The shape of the curves conforms to the one expected for systems with a percolation threshold. An example is the grain consolidation model with random packings [1]. For small values of $L$, however, the shape 
of the local percolation probabilities resembles that for the central pore model [1]. All of these conclusions are very preliminary. More work is necessary to corroborate them, and to validate the measurement.

ACKNOWLEDGEMENT: The authors are grateful to Chr. Ostertag-Henning, E. Haslund, Dr. U. Mann, Prof.Dr. B. Nøst, Prof.Dr. D.H. Welte and Prof.Dr. R. Koch for discussions, and to Norges Forskningsråd and the Forschungszentrum Jülich for partial financial support. 


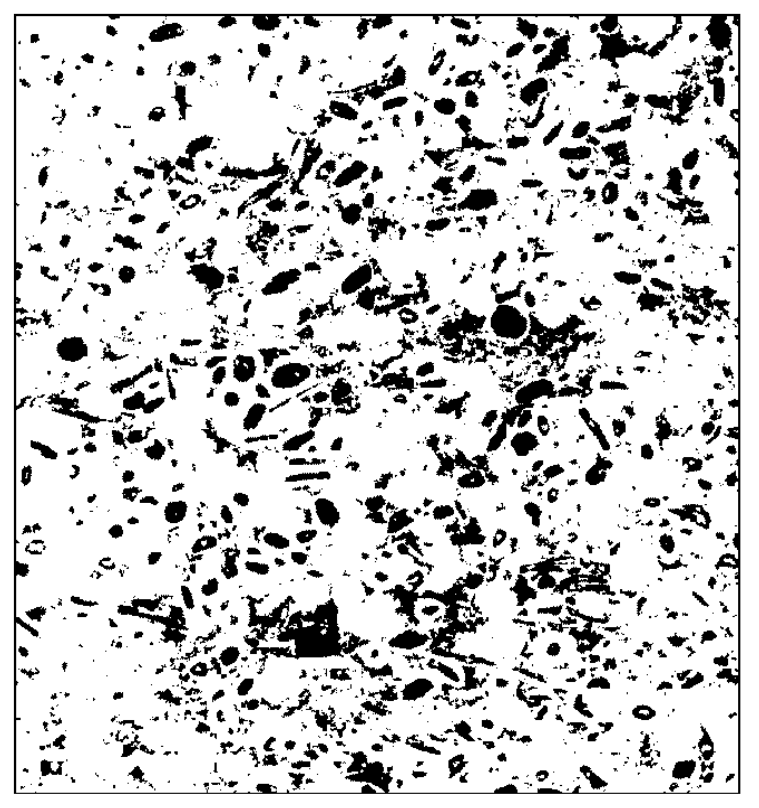

FIG. 1. Digitized thin section image of Savonnier oolithic sandstone [13]. The pore space $\mathbb{P}$ is coloured black, the matrix space is rendered white. 


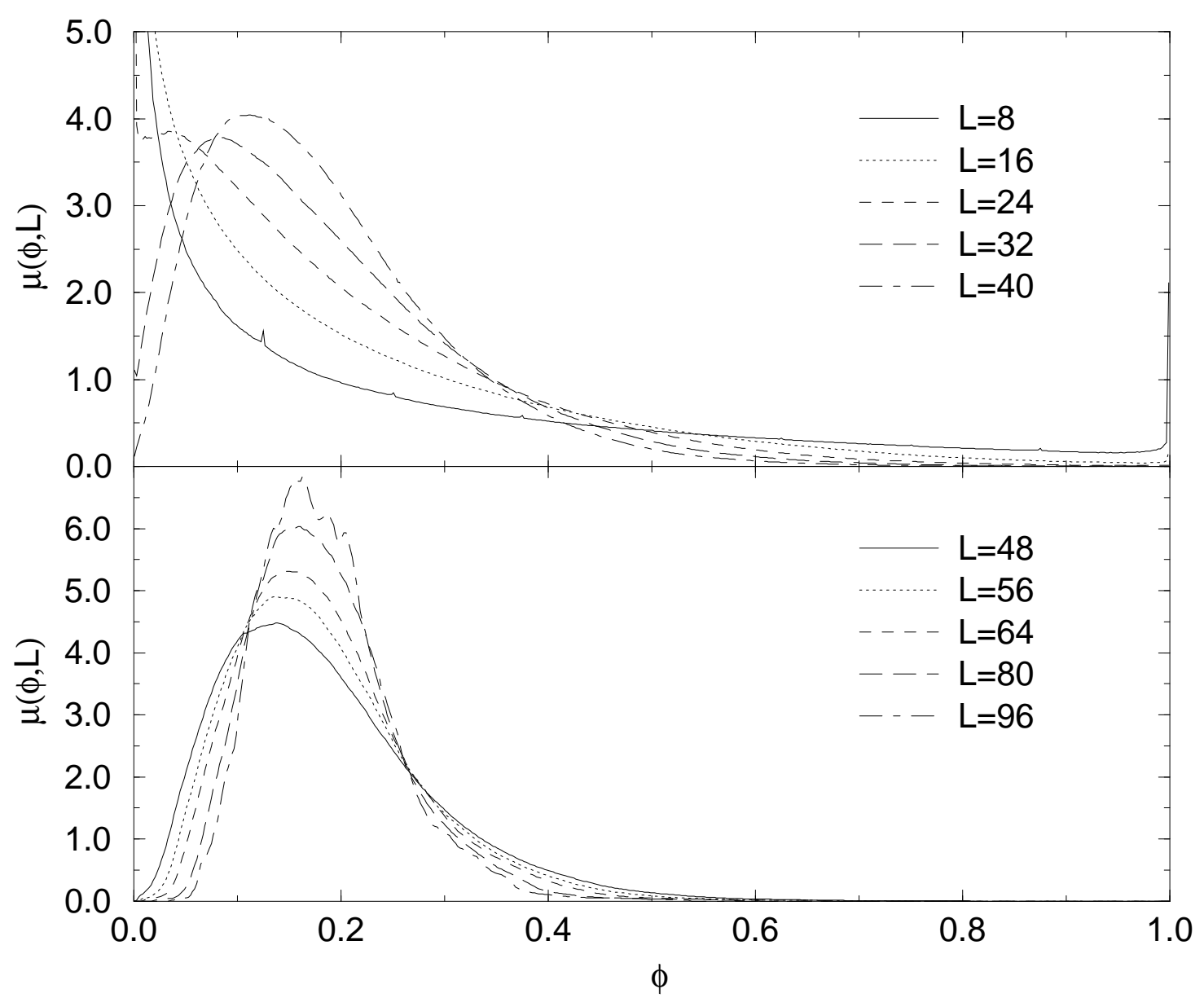

FIG. 2. Local porosity distributions $\mu(\phi ; L)$ for a cubic measurement cells of sidelengths $L$. The values of $L$ for the different curves are indicated in the legend. 


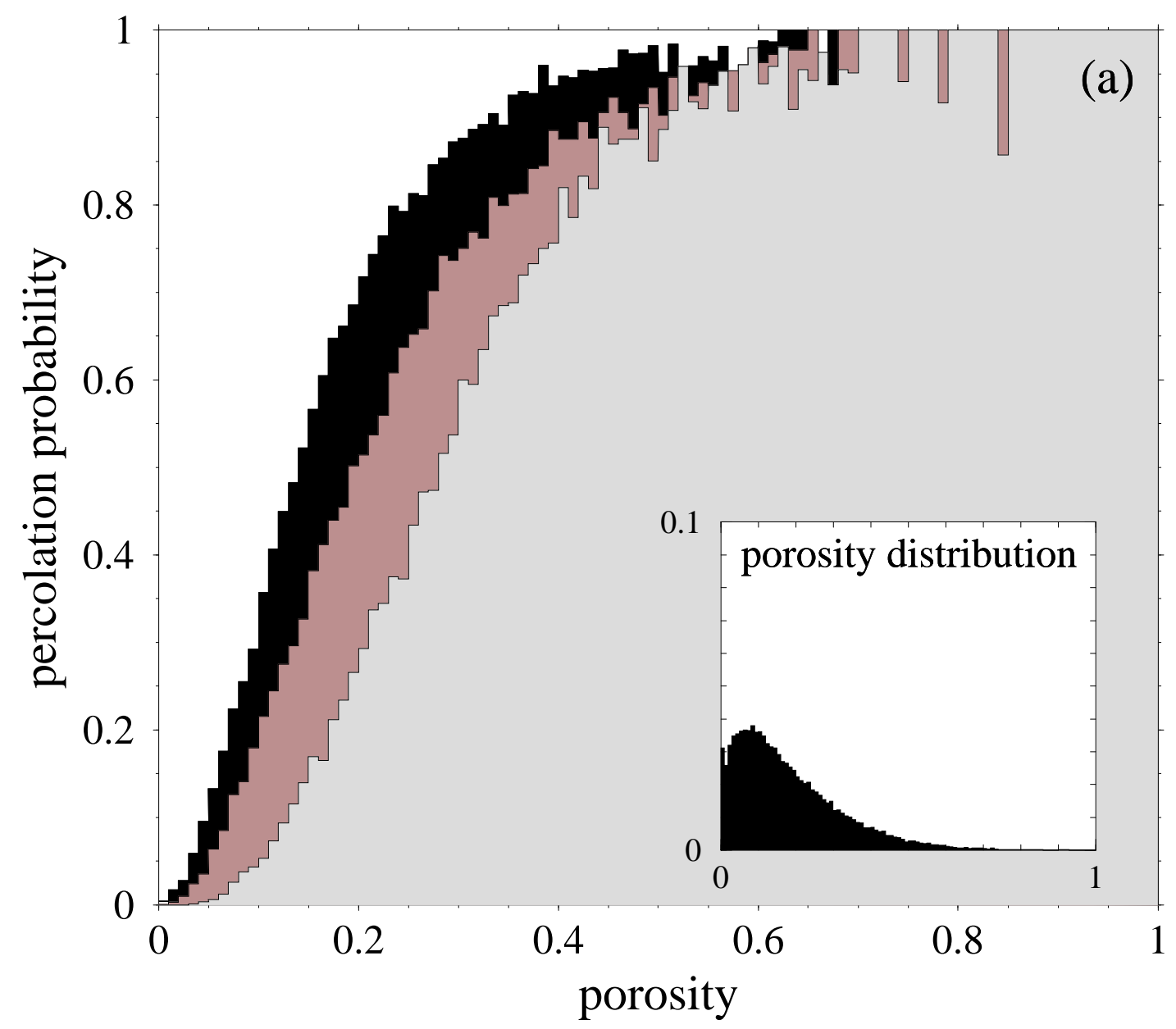

FIG. 3. Local percolation probability functions $\lambda_{x}(\phi, L)$ (black), $\lambda_{y}(\phi, L)$ (medium gray), and $\lambda_{z}(\phi, L)$ (light gray) for the Savonnier oolithic sandstone shown in Figure 11 using a simple cubic lattice of measurement cells with side lengths (lattice constant) $L=32$. The local porosity distribution $\mu(\phi, L)$ for $L=32$ is shown as the inset. 


\section{REFERENCES}

[1] R. Hilfer, "Geometric and dielectric characterization of porous media," Phys. Rev. B, vol. 44, p. 60, 1991.

[2] R. Hilfer, "Local porosity theory for flow in porous media," Phys. Rev. B, vol. 45, p. $7115,1992$.

[3] R. Hilfer, "Geometry, dielectric response and scaling in porous media," Physica Scripta, vol. T44, p. 51, 1992.

[4] F. Boger, J. Feder, R. Hilfer, and T. Jøssang, "Microstructural sensitivity of local porosity distributions," Physica A, vol. 187, p. 55, 1992.

[5] R. Hilfer, "Local porosity theory for electrical and hydrodynamical transport through porous media," Physica A, vol. 194, p. 406, 1993.

[6] B. Hansen, E. Haslund, R. Hilfer, and B. Nøst, "Dielectric dispersion measurements of salt water saturated porous glass compared with local porosity theory," Mater.Res.Soc.Proc., vol. 290, p. 185, 1993.

[7] R. Hilfer, B.Nøst, E.Haslund, Th.Kautzsch, B.Virgin, and B.D.Hansen, "Local porosity theory for the frequency dependent dielectric function of porous rocks and polymer blends," Physica A, vol. 207, p. 19, 1994.

[8] E. Haslund, B. Hansen, R. Hilfer, and B. Nøst, "Measurement of local porosities and dielectric dispersion for a water saturated porous medium," J. Appl. Phys., vol. 76, p. 5473, 1994.

[9] R. Hilfer, "Probabilistic methods, upscaling and fractal statistics in porous media," $Z$. Blatt f. Geol. Paläont., p. in print, 1996.

[10] R. Hilfer, "Transport and relaxation phenomena in porous media," Advances in Chemical Physics, vol. XCII, p. 299, 1996. 
[11] B. Nøst, B. Hansen, and E. Haslund, "Dielectric dispersion of composite materials," Physica Scripta, vol. T44, p. 67, 1992.

[12] C. Ostertag-Henning, "Modellierung von Porosität und Permeabilität in Realgesteinen anhand der lokalen Porositätstheorie," tech. rep., Institut für Paläontologie, Universität Erlangen, Germany, 1995.

[13] C. Ostertag-Henning, B. Virgin, T. Rage, R. Hilfer, R. Koch, and U. Mann, "Messung dreidimensionaler lokaler Porositätsverteilungen," 1995. to be published. 